\title{
UNIFIED METHODOLOGY FOR CLOUD STORAGE PROVIDERS' APIS AND PERFORMANCE ANALYSIS FOR EFFECTIVENESS OF THE APPROACH
}

\author{
Hira Zahid ${ }^{1}$, Muazzam A. Khan², Muhammad Usman Akram¹, Abdul Wahid ${ }^{2}$ and Asad W. Malik ${ }^{2}$ \\ ${ }^{I}$ Department of Computer and Software Engineering, CEME \\ ${ }^{2}$ Department of Computing, SEECS \\ National University of Sciences and Technology, Islamabad, Pakistan
}

\begin{abstract}
Many cloud users do not register for the premium plans to get unlimited access to their storage services. Consequently, they have limited storage. In this study, we design and implement a unified API for cloud storage providers i.e. Dropbox, Google Drive and One Drive to overcome the issue of limited storage. Our study is based on two experiments: web app development and the development of core API. In web app, we developed a simple web app that will be connected to all three cloud storage providers. In the core API, we developed a core API enabling the storage functionality in all the three drives. Both methodologies enable us uploading of the files on all these drives or any one of them. In case one service provider's server is down, we can upload and retrieve our files from the other storage service provider available via our unified API. PHP core language is used to develop the unified API. The results of our approach are justified using the most accurate and open source tool J-meter. We have compared both methodologies and the results shows that both perform well in terms of latency and throughput when compared to related work in the literature. Furthermore, we also highlight some future directions regarding such research.
\end{abstract}

\section{KEYWORDS}

Cloud API, Unified Cloud, Unified API, Drop Box API, Google Drive API, PAAS API, SAAS API

\section{INTRODUCTION}

In this modern era, cloud computing is becoming highly dependent upon APIs in order to enhance the cloud experience and to achieve cross compatibility for cloud environments. In this section, we described cloud storage APIs in detail.

\subsection{Cloud Storage Providers' APIs Overview}

APIs in cloud are categorized into the following three groups ${ }^{1}$.

\subsubsection{Google Storage API}

Google gives the facility of two APIs in order to store cloud oriented data and information. It includes an API in JSON format i.e. Google cloud storage JSON format and there is another API which is known as application development interface and it is in the format of XML API.

\subsubsection{Dropbox API}

Dropbox has large amount of SDKs available in order to make the integration of its services and platforms with third party applications. It provides the development kit in different languages for the applications. When developers add the call function to Dropbox API then the client authentication is required at a specific level by every application. If there is some programmer who is making requests by using SDKs of any company then the Development Kit is responsible for the details. If developer tends to utilize the drop box API through the calls that are relied on common method of HTTP then there is a need to apply for the right type of authentication for every end point.

\footnotetext{
${ }^{1}$ See https://bbvaopen4u.com/en/actualidad/cloud-computing-without-apis-possible
} 


\subsubsection{One Drive API}

The One drive provide the REST API which is utilizing a portion of Graph API of Microsoft that enable the application in order to connect with the content that is stored in one drive and share point. The API of One drive is shared among one drive, one drive for the solution of business, libraries of share point documents and the office document in order to enable the flexibility of reading and storing the content in any of these platforms with the one code.

A small survey was conducted on social media platform before taking these cloud storage providers API to be studied into this study. It is concluded that mostly people either general public or researchers are aware of these three platforms.

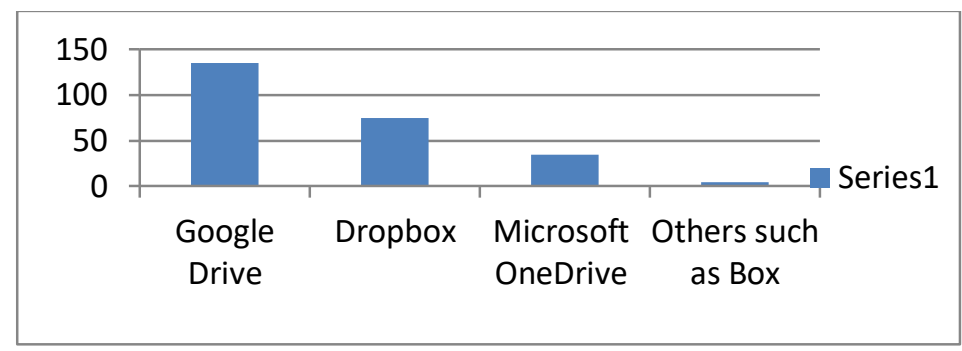

Figure 1. Number of people who are aware off cloud storage providers

\section{LITERATURE REVIEW}

Yang, Chang, and Yuan (2015) proposed a cloud storage access gateway based on CDMI-compliant to solve the different integration methods of Dropbox API, Google drive API and one drive API. The proposed cloud storage access gateway enable web service providers (WSP) to easily develop services of web. Consequently, this method allows to access data of users in their cloud storages and users can easily handle their data in their respective cloud storage services. The software developers and WSP can manage data on cloud storage using the proposed access gateway, hence, they can save their time and cost in learning different APIs.

Jiang, Yu, and Huang (2016) proposed an approach named as Cloud Days where they have devised a concept of merging different cloud storages into a single cloud storage account. This application follows the concept of RAID 0 that helps to increase the performance, security level and capacity of storage. It also helps in minimizing the total file downloading OR uploading time to/from the cloud. Different experiments were conducted using their proposed application and the proposed approach tested on drop box and thus this study shows that many storages of cloud can be merged together. The proposed application also reduced the time of file uploading instead of uploading a file to drop box website.

Silva, Costa, and Oliveira (2013) provide a cross platform interface by using REST oriented API. File based on Meta and their permissions are handled by token mechanisms of HTTP and database relying on NOSQL. The system is designed to fulfill the requirements for deep and vast number of cloud storage tenants. The reliability, performance and availability of the system was verified and validated by technology tests on the data of user in the real time environment.

$\mathrm{Wu}$ and Lee (2013) proposed a platform that provides support to applications for interoperation with different cloud providers' services by using a standardized interface. This proposed approach basically provides a common API that decreases the existing shortfall of cloud standardization and gives safe and redundant allocation of services. Authors also proposed an idea to combine the services from different cloud providers and then to decorate it with additional functions like for example ciphering on the fly and redundancy.

Shah, Nair, and Thakar (2014) proposed the design of API access control mechanism and then implemented it by using OAuth that tends to simplify the flow of process under several scenarios of applications. Authors also illustrated that how their access control mechanism can be taken as RESTful web services. The proposed design gives a solution of scalable system architecture to assist several behaviors of cloud API. 


\section{METHODOLOGY}

This research is based on implementing the most commonly used cloud storage providers i.e. drop box, google drive and one drive (Gessert, Friedrich, Wingerath, Schaarschmidt, \& Ritter, 2014). The main aim is to provide a unified service or a unified web API that enable the uploading of the files in all these drives via unified API (Sellami, Bhiri, \& Defude, 2014).

We have implemented this project in core PHP language since PHP is an open source and an easy language. All these providers have their documentation available in PHP language ${ }^{2}$.

We have divided out proposed methodology in the following three main phases:

A. Authentication: User will authenticate himself and cloud storage provider will assign a specific access token to that user which will be used further to make integration with that specific cloud storage provider.

B. Token saving after authentication: Every time access token is changed randomly, in order to make successful integration we store the access token in our local database so that it can be accessed from there for integration purpose.

C. Uploading file after authentication: After the completion of authorization phase, the file will be uploaded on any cloud storage provider.

In case when user skip step 2, every time there is a need to make sign in from every cloud storage provider i.e. Google drive, Dropbox and One drive.

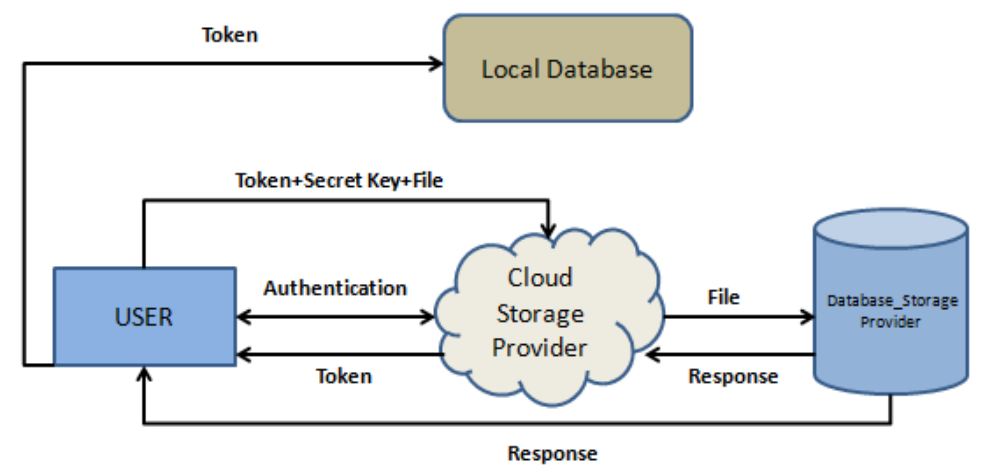

Figure 2. Process Overview for every cloud storage provider's API

In Figure 2, there is a centralized cloud storage provider for which user will be getting signed in for authentication. After authentication, a user will be assigned a secret key and an access token. The access token will also be stored in local database to be fetched later. When a user upload the file via unified interface the access token will be taken from local database and the file will also be uploaded by the authenticated user. Then after uploading, a response will be generated from the database storage provider.

\subsection{Methodology of Web APP Integrating Storage Providers}

In this methodology, a simple web app concept is used, also uploading function of all the three cloud storage providers are embedded in the web app code.

This experimentation is based on developing a web app in core PHP as follows:

\footnotetext{
${ }^{2}$ See:

Dropbox. (2018). Developers - Dropbox. [online]Available at: https://www.dropbox.com/developers/ [Accessed 20 Jan. 2018].

Apps.dev.microsoft.com. (2018). [online] Available at: https://apps.dev.microsoft.com [Accessed 20 Jan. 2018].
} 


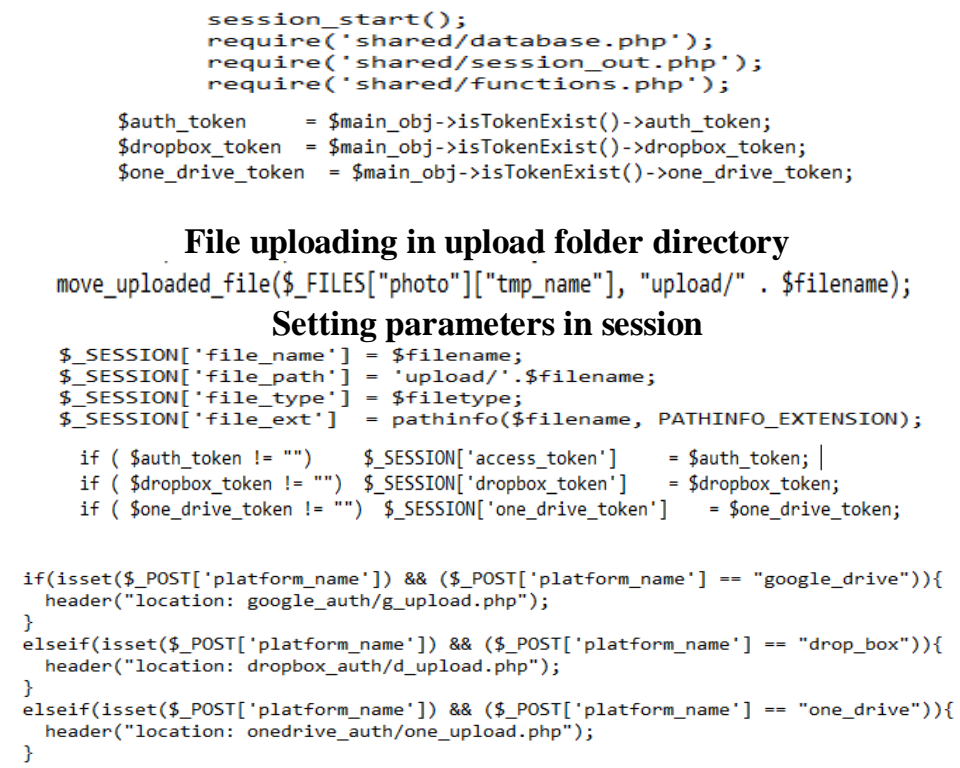

The above code is providing a unified interface that enable the uploading the files on all the drives.

\subsection{Setting Parameters in J-meter for testing Unified web APP}

The unified web app project is recorded using J-meter recording feature. J-meter automatically records the accurate parameters and the file uploading path, also J-meter store it in test plan. Now, the below test plan is ready to be tested.

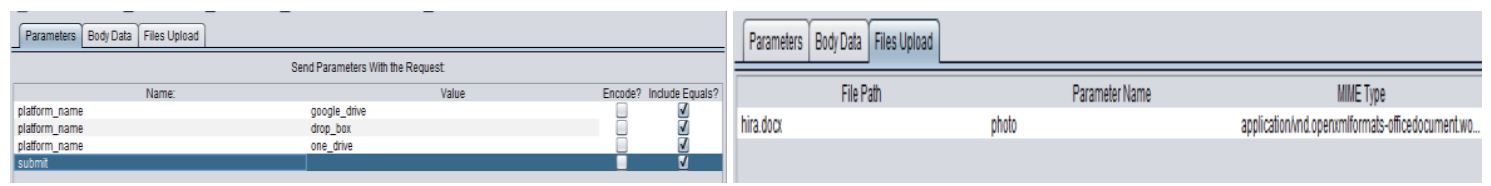

Figure 3. Parameters setting on recording the scenario

J-meter also recorded the file uploading behavior and stores the file in the test plan as it is done in the browser. It is well depicted in the above Figure 3.

\subsection{Novelty of our Proposed Solution of Unified API}

An API is supposed to work in a plug and play mode i.e. one can use it in any application. Therefore, it is better to separate the API logic and make the approach more object-oriented and modular. Using API instead of SDK gives us a lot more flexibility and control. Moreover, we can get rid of all the intense, unnecessary SDK files and it enable us to just implement the features we need. In other words, the proposed API is adopting the architecture of switching mechanism i.e. plug and play. API switching is done using the toggle function. All the APIs are initialized in the start then they are toggled as ON and OFF. When an API is toggled, the functions such as upload file or add access token don't touch the API of that particular API Platform.

In this research, instead of using the concept of SDKs, access points of the cloud platforms are used directly. Usually API does not provide the functionality of storage, however, our proposed API provides the functionality of storage. The below class diagram is well illustrating the proposed unified web API concept. 


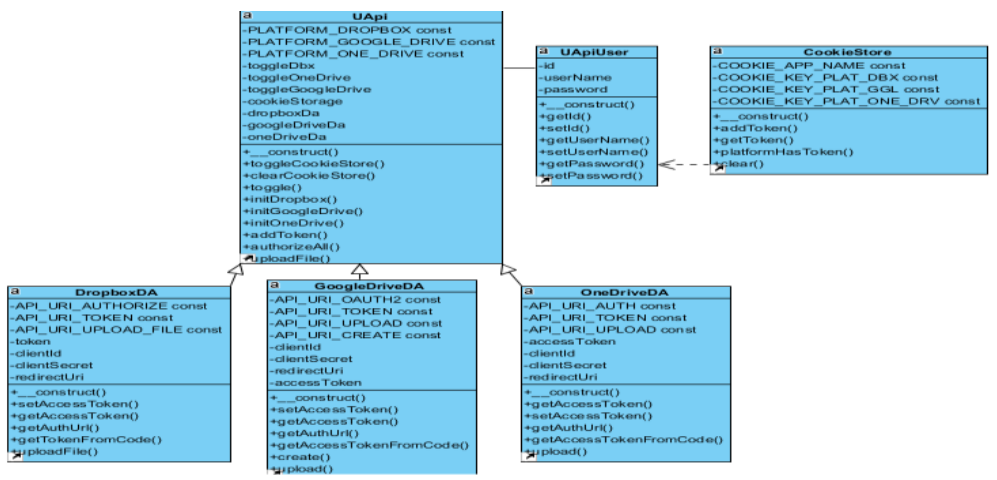

Figure 4.Class Diagram for Unified API (Cookie Methodology)

In order to improve this methodology, cookies are used to store the access token. In the authentication process, these cloud platforms create an app folder in user account. this way we are storing the token in their access points with the help of cookies since every browser has its own cookie information. When the access point will be accessed, the cookie information will be forwarded as well to that access point. Cookies are supposed to be secure by browser since they are encrypted by browsers. Access tokens are the temporary data, therefore, storing it in cookies is a best approach.

\subsection{Parameters Setting in J-meter for Performance Measures}

J-meter sets the parameters for the unified API methodology. The parameters are set by J-meter by utilizing the feature of recording scenario. In this methodology, all the drives toggle function is ON. The testing tool recorded the http request and parameters exactly as they are.

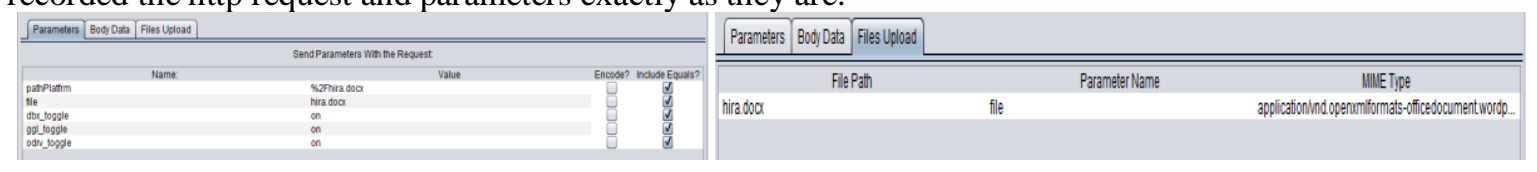

Figure 5.Parameters being recorded

\section{PERFORMANCE EVALUATION}

After implementing the unified API code we successfully enable uploading of the file in all the drives. First we go through the authentication process in which a user needs to make himself registered and have to sign in from one interface instead of signing in different cloud storage providers.

User either checks any one of these platforms OR checks all platforms to upload the file. In case of unavailability of any provider, the file will be uploaded on the other platform. Through this way a user will not need to visit again another website and login there. He can upload his file into another cloud storage provider staying on the same interface rather than switching into another website or interface.

\subsection{Web App Methodology}

Figure 6 shows that the unified web app is giving the quite good throughput result i.e. 432 per minute but there is high deviation. 


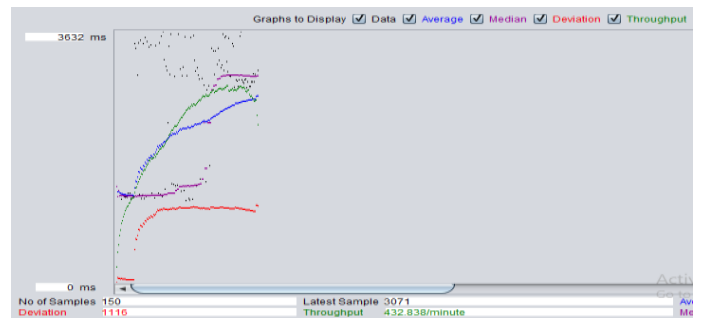

Figure 6. Throughput and deviation Analysis

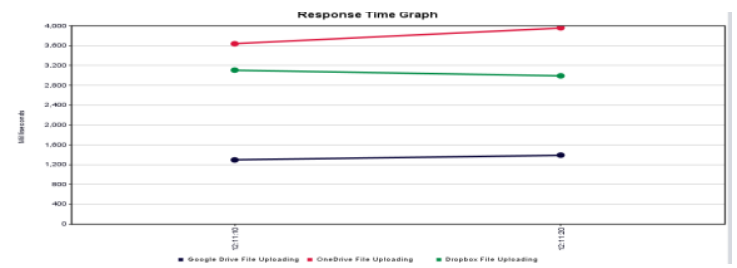

Figure 7. Response time Analysis for all drives in unified app

Figure 7 shows that good response time is achieved on setting 50 concurrent users for every drive. It is also depicted that Google drive and Dropbox APIs for file uploading are more efficient as compared to One drive.

\subsection{Unified API Methodology}

In our proposed methodology cookies are used for storing access token. An API is developed in core PHP utilizing the concept of toggling.

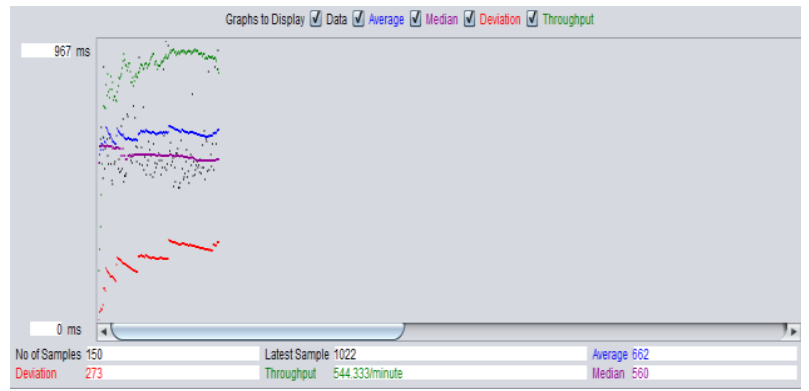

Figure 8. Throughput and Average Analysis

Figure 8 shows that we are achieving great throughput and less deviation whereas in the previous studies such results were not clear.

\subsubsection{Performance Analysis with Respect to Individual Drive in Cookie Methodology}

Figure 9 shows the response time for Google drive that seems a very good response time using our unified API methodology. On setting only Google drive toggle function switching to ON position, it gives good response time even setting more concurrent users i.e. 150.

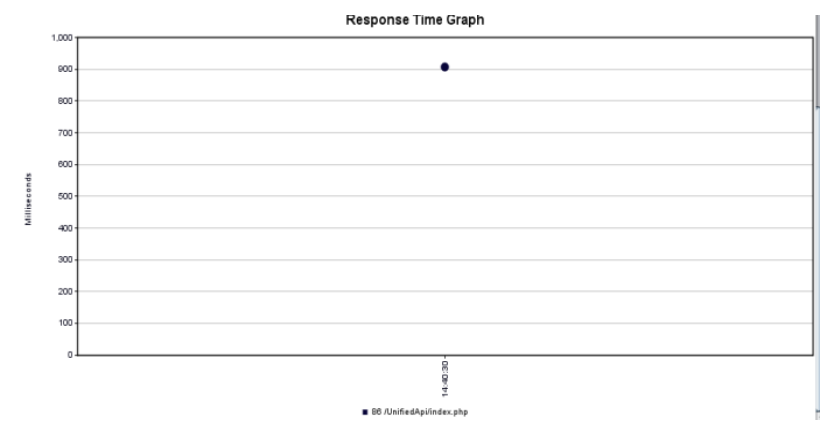

Figure 9.Response time for google drive file uploading 


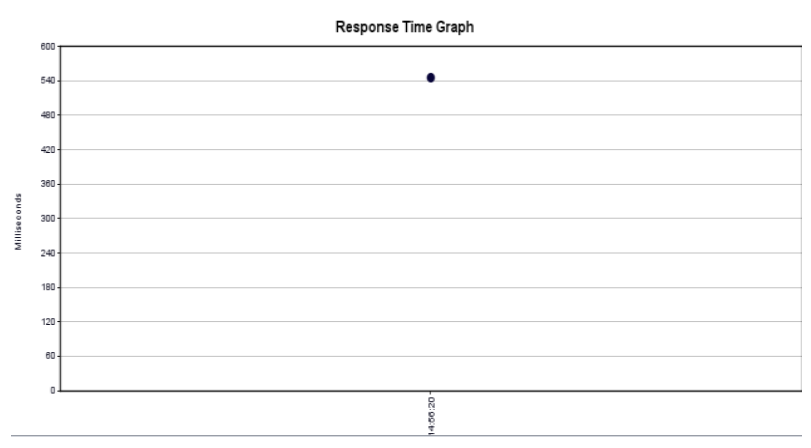

Figure 10. Response time for dropbox in cookie methodology

Figure 10 shows the response time for Dropbox, similar to Google drive, it is observed that Dropbox is having a good response time. On setting the only Dropbox toggle function to ON position it gives the good response time even setting more concurrent users i.e. 150. In web app methodology, quite high response time is appearing with 50 concurrent users.

\subsection{Cloud Storage Access Gateway Average Latency (Uploading Files)}

In this section, we present and compare our proposed scheme with HTTP API and cloud Elements API. The HTTP API and Cloud Element API have high average latency as compared to our proposed Unified API approach as shown in Figure 11.

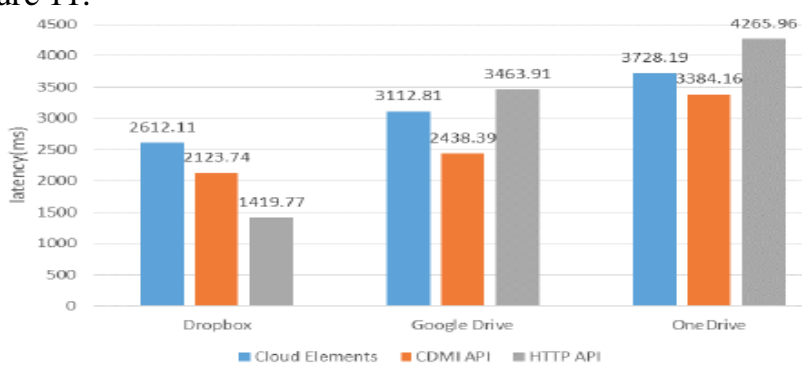

Figure 11. Results of Uploading Files

\subsection{Unified Web API Average Latency (Uploading Files)}

Figure 12 clearly depicts that when we take high sample time i.e. 1298, our proposed scheme have lower latency. The latency is estimated for the 50 samples and all samples have range of 1200 (ms) latency. On calculating the average as below:

$1298(\mathrm{~ms}) * 50$ samples $=64,900$

$64,900 / 50=\underline{\mathbf{1 , 2 9 8}(\mathbf{m s}) .}$

In the literature work comparison review, quite high latency (ms) is observed in uploading files, however, our unified API approach is having lower latency because of accessing the direct access points of the providers by using CURL calls. Instead of utilizing the concept of SDKs, access points of the cloud platforms are utilized directly. Cookies are used in our methodology for storing access token. Direct access to the providers is established using CURL operations. Lower latency is achieved even implementing it using unified API approach.

This study is a primary foundation to expand this storage system in such a way that we may retrieve or download our files in a unified storage system and to make analysis of this storage system with respect to big data.

On the other hand this proposed solution is only analyzed regarding performance and is not analyzed with respect to security aspects. 


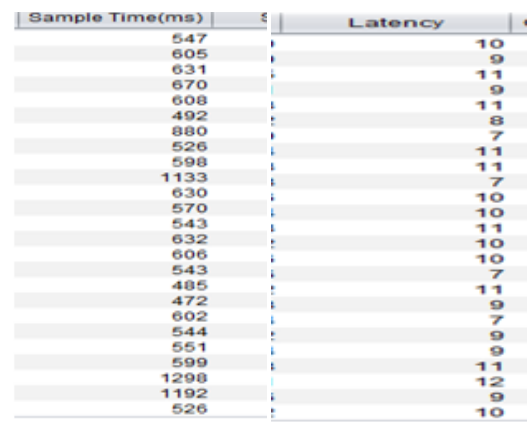

Figure 12. Results of Uploading Files

\section{CONCLUSION}

It is time consuming when we upload our data by signing into different cloud storage providers. Therefore, in this work, we try to achieve the objective of time saving by implementing a unified web API and web app methodology. In our study, two experiments were conducted for cloud storage providers' integration. The first experiment of web app has quite high latency and high deviation. The second experiment of unified API has better results with respect to performance such as lower latency, high throughput and lower deviation. We used direct access points of the providers using the CURL calls and thus it is leading to the better performance. In web app methodology, we faced high deviation and quite high latency as compared to the unified API methodology. The unified web API also showed better performance in terms of latency when it is compared to similar work from the literature (Yang, Chang, \& Yuan, 2015).

In a nutshell, it is better to utilize the concept of unified web API methodology of this study instead of using the SDK files or developing a simple web app. In future, we aim to make advancement in upload service in such a way that when a user upload the file in a specific drive via unified API it automatically creates a backup or copy of the file in all remaining drives instead of checking all the boxes. Moreover, which framework will go best for this unified API for cloud storage providers will also be included in our future research.

\section{REFERENCES}

Silva, L. A. B., Costa, C., \& Oliveira, J. L. (2013). A common API for delivering services over multi-vendor cloud resources. Journal of Systems and Software, 86(9), 2309-2317.

Wu, G., Liu, F., Tang, H., Huang, K., Zhang, Q., Li, Z., .. \& Jin, H. (2016, June). On the performance of cloud storage applications with global measurement. In 2016 IEEE/ACM 24th International Symposium on Quality of Service $(I W Q o S)$ (pp. 1-10). IEEE.

Gessert, F., Friedrich, S., Wingerath, W., Schaarschmidt, M., \& Ritter, N. (2014). Towards a Scalable and Unified REST API for Cloud Data Stores. In GI-Jahrestagung (pp. 723-734).

Wu, M. Y., \& Lee, T. H. (2013, April). Design and implementation of cloud API access control based on OAuth. In IEEE 2013 Tencon-Spring (pp. 485-489). IEEE.

Sellami, R., Bhiri, S., \& Defude, B. (2014, June). ODBAPI: a unified REST API for relational and NoSQL data stores. In 2014 IEEE International Congress on Big Data (pp. 653-660). IEEE.

Shah, S., Nair, A., \& Thakar, U. (2014, December). A framework to integrate multiple cloud storage services and provide consistent feature set. In 2014 Annual IEEE India Conference (INDICON) (pp. 1-5). IEEE.

Yang, Z. Y., Chang, C. P., \& Yuan, S. M. (2015, December). Cloud Storage Access Gateway. In 2015 IEEE International Conference on Smart City/SocialCom/SustainCom (SmartCity) (pp. 1143-1146). IEEE.

Jiang, H., Yu, Q., \& Huang, K. (2016, August). Design and implementation of an improved cloud storage system. In 2016 12th International Conference on Natural Computation, Fuzzy Systems and Knowledge Discovery (ICNC-FSKD) (pp. 1816-1823). IEEE.

Mishra, B., \& Jena, D. (2016, October). Securing files in the cloud. In 2016 IEEE International Conference on Cloud Computing in Emerging Markets (CCEM) (pp. 40-45). IEEE. Apps.dev.microsoft.com. (2018). [online] Available at: https://apps.dev.microsoft.com [Accessed 20 Jan. 2018]. 